\title{
Erratum: "Aluminohydrides:
} Structures, NMR, Solid-State Reactions"

[Russian Journal of Inorganic Chemistry 53 (13), 2048 (2008)]

V. P. Tarasov and G. A. Kirakosyan

Kurnakov Institute of General and Inorganic Chemistry, Russian Academy of Sciences,

Leninskii pr. 31, Moscow, 119991 Russia

E-mail: tarasov@igic.ras.ru

DOI: $10.1134 / \mathrm{S} 0036023609080269$

Equations (6) and (7) on p. 2063 should be read as follows

$$
e q=(1-\gamma) e q^{\text {lat }}\left[\mathrm{V} / \mathrm{cm}^{2}\right]=41.37 \times 10^{18} \chi^{Q}[\mathrm{MHz}] / \mathrm{Q}[\text { barn }]
$$

and

$$
e q=e q^{\mathrm{val}}\left[\mathrm{V} / \mathrm{cm}^{2}\right]=41.37 \times 10^{18} \chi^{Q}[\mathrm{MHz}] / \mathrm{Q}[\text { barn }] .
$$

